\title{
ON RELATIONSHIPS BETWEEN NÖRLUND MEANS FOR DOUBLE SERIES ${ }^{1}$
}

\author{
CHARLES N. MOORE
}

The purpose of the present paper is to generalize to double series certain theorems concerning relationships between Nörlund means for single series established by Nörlund ${ }^{2}$ and Marcel Riesz. ${ }^{3}$ We consider two infinite sequences of non-negative numbers $p_{i j}, q_{i j}$, and we set

$$
\begin{aligned}
P_{m n} & =\sum_{i=0, j=0}^{m, n} p_{i j}, \quad Q_{m n}=\sum_{i=0, j=0}^{m, n} q_{i j}, \\
N_{m n}^{(p)}(s) & =\frac{\sum_{i=0, j=0}^{m, n} p_{m-i, n-j} s_{i j}}{P_{m n}} \quad(\text { and } p, P \text { replaced by } q, Q),
\end{aligned}
$$

where the $s_{i j}$ are the partial sums of a double series $\sum u_{i j}$, which remain bounded for all $(i, j)$.

The necessary and sufficient conditions for regularity of Nörlund means in the case of such double series have been given by $\mathrm{C} . \mathrm{N}$. Moore in Theorem II of Chapter II of his book ${ }^{4}$ entitled Summable series and convergence factors which will be subsequently referred to as S.S.C.F. In the case where the weights $p_{i j}$ are non-negative real numbers, condition (10) of that theorem is obviously satisfied. Condition (11) takes the form

$$
\lim _{m, n \rightarrow \infty} \frac{\sum_{j=0}^{n} p_{m-i, n-i}}{P_{m n}}=0, \quad \lim _{m, n \rightarrow \infty} \frac{\sum_{i=0}^{m} p_{m-i, n-j}}{P_{m n}}=0
$$

where $0 \leqq i \leqq m-k$ in the first equation and $0 \leqq j \leqq n-l$ in the second one, $k$ and $l$ being any fixed integers $>0.5$

Presented to the Society, December 29, 1953; received by the editors January 7, 1954 and, in revised form, March 22, 1954.

1 This research was supported by the United States Air Force through the Office of Scientific Research of the Air Research and Development Command, under Contract No. AF 18(600)-691.

${ }^{2}$ Sur une application des fonctions permutables, Lunds Universitets Arsskrift, Avdelning 2, vol. 16 (1919). Cf. also Hardy, Divergent series, Chapter IV.

3 Proc. London. Math. Soc. (2) vol. 22 (1924) pp. 412-419. Cf. also Hardy, loc. cit.

- Vol. XXII, Amer. Math. Soc. Colloquium Publications.

${ }^{5}$ We have changed the notation used in S.S.C.F., which was somewhat confusing in that $m$ and $n$ were employed in two different senses in the same formula. 
We next prove the consistency of any two regular Nörlund methods where each row and each column of the infinite matrix $\left(p_{m n}\right)$ furnishes a regular Nörlund mean for simple sequences. Given two such methods $\left(N, p_{m n}\right)$ and $\left(N, q_{m n}\right)$, we define a third method $\left(N, r_{m n}\right)$ by the equation

$$
r_{m n}=\sum_{i=0, j=0}^{m, n} p_{i j} q_{m-i, n-j}
$$

We then readily obtain

$$
N_{m n}^{(r)}(s)=\sum_{i=0, j=0}^{m, n} \gamma_{m n i j} N_{i j}^{(q)}(s)
$$

where

$$
\gamma_{m n i j}=p_{m-i, n-j} Q_{i j} / \sum_{\mu=0, \nu=0}^{m, n} p_{m-\mu, n-\nu} Q_{\mu \nu}
$$

The necessary and sufficient conditions that the transformation defined by (5) and (6) will be regular have been given in Theorem VI of Chapter I of S.S.C.F. In the present case it is readily seen that conditions $\left(\mathrm{A}^{*}\right)_{2},\left(\mathrm{~A}_{1}^{*}\right)_{2}$, and $\left(\mathrm{C}^{*}\right)_{2}$ are satisfied. To show that $\left(\mathrm{E}_{1}^{*}\right)$ and $\left(\mathrm{E}_{2}^{*}\right)$ hold we must prove that

(7) $\lim _{m, n \rightarrow \infty} \sum_{j=0}^{n} \gamma_{m n i j}=0=\lim _{m, n \rightarrow \infty} \sum_{i=0}^{m} \gamma_{m n i j} \quad(0 \leqq i<m ; 0 \leqq j<n)$.

The proofs of the two equalities in (7) are similar, so we shall consider only the first. Since $P$ and $Q$ are regular, we have from conditions (3)

$$
\lim _{m, n \rightarrow \infty} \frac{Q_{m-i, n}}{Q_{m n}}=0=\lim _{m, n \rightarrow \infty} \frac{Q_{m, n-i}}{Q_{m n}},
$$

and analogous conditions for $P$, where $0<i \leqq m, 0<j \leqq n$, and $(m-i)$ and $(n-j)$ remain fixed while $m$ and $n \rightarrow \infty$. But we have, since $Q_{i j}$ is a monotonic increasing function of $i$ and $j$, satisfying (8),

$$
\begin{aligned}
& 0<p_{m-i, n-j} Q_{i j} / \sum_{h=\mu}^{m} p_{m-h, n-j} Q_{h j}<\frac{p_{m-i, n-j}}{\sum_{h=\mu}^{m} p_{m-h, n-j}} \cdot \epsilon \\
&\left(m \geqq \mu \geqq m_{1} ; \mu>i ; n \geqq j \geqq n_{1}\right),
\end{aligned}
$$

where $\epsilon$ is a given positive quantity and $\mu, m$, and $n$, are chosen ac- 
cordingly. We have a similar inequality for each $j$ such that $n_{1}<j \leqq n$. Also, since each column of the matrix for $P$ furnishes a regular transformation for a simple sequence, we have a similar inequality for $0 \leqq j \leqq n_{1}, m \geqq m_{2}$, if we properly choose an $m_{2} \geqq m_{1}$. Combining all these inequalities, we obtain

$$
\begin{aligned}
0 \leqq \sum_{j=0}^{n} \gamma_{m n i j} & =\frac{\sum_{j=0}^{n} p_{m-i, n-j} Q_{i j}}{\sum_{i=0, j=0}^{m, n} p_{m-i, n-j} Q_{i j}} \\
& \leqq \frac{\sum_{j=0}^{n} p_{m-i, n-j} Q_{i j}}{\sum_{h=\mu, j=0}^{m, n} p_{m-h, n-j} Q_{i j}}<\epsilon \quad\left(m \geqq m_{2}, n \geqq n_{1} ; m>i\right) .
\end{aligned}
$$

Thus we have established the first equality in (7), and as noted above, the proof of the second equality is similar. Hence the regularity of the transformation defined by (5) and (6) is established.

The regularity of this transformation enables us to infer that $s_{m n} \rightarrow s^{\prime}\left(N, q_{m n}\right)$ implies $s_{m n} \rightarrow s^{\prime}\left(N, r_{m n}\right)$. Similarly we can show that $s_{m n} \rightarrow s\left(N, p_{m n}\right)$ implies $s_{m n} \rightarrow s\left(N, r_{m n}\right)$. Thus we have the theorem:

Theorem I. Any two regular Nörlund methods for summing double series where each row and each column furnishes a regular method for simple series are consistent.

In view of the supplementary conditions on the Nörlund matrix which we found it necessary to impose in order to prove Theorem I, we are led to ask two questions. First, are all regular Nörlund matrices for double series such that the transformations furnished by the individual rows and columns are likewise regular transformations? In case this is not so, are the supplementary conditions in Theorem I due to the manner of proof, and not inherently necessary? We shall show by examples that the answers to both questions are in the negative.

Let $k$ be an integer $>1$ and consider the double matrix where

$$
\begin{aligned}
& p_{0 j}=1 \quad(j=0,1,2, \cdots, 2 k-1), \quad p_{0,2 k}=2 k, \\
& p_{0 j}=1 \quad\left(j=2 k+1, \cdots, 2 k^{2}-2 k\right), \quad p_{0,2 k^{2}-2 k+1}=2 k^{2},
\end{aligned}
$$

with further $p_{0 j}$ defined in analogous fashion. We further define

$$
p_{1 j}=1
$$


The columns with even indices are defined in the same manner as the $p_{0 j}$ and the columns with odd indices in the same manner as the $p_{1 j}$. It is then readily seen that the columns with even indices do not furnish a regular transformation for simple series, whereas the double matrix gives a regular transformation for double series.

We now apply the transformation defined above to the double series:

$$
\begin{array}{r}
1-1+1-1+\cdots, \\
-1+1-1+1-\cdots,
\end{array}
$$

The partial sums for this double series gives us the sequence:

$$
\begin{array}{lllll}
1 & 0 & 1 & 0 & \cdots \\
0 & 1 & 0 & 1 & \cdots
\end{array},
$$

If we form the transformation for the sequence containing $2 k^{r}$ rows and $2 m$ columns, we obtain

$\sigma_{4 m k^{r}}=\left[4 m\left(k^{r+2}-k\right) /(k-1)+m r / 2\right] /\left[6 m\left(k^{r+2}-k\right) /(k-1)+m r\right]$.

If we allow $m$ and $r$ to become infinite, we see that

$$
\sigma_{4 m k^{r}} \rightarrow 2 / 3 \text {. }
$$

Suppose now that we interchange the definitions of odd and even columns in the double Nörlund matrix. Then we have

$\sigma_{4 m k^{r}}=\left[2 m\left(k^{r+2}-k\right) /(k-1)+m r / 2\right] /\left[6 m\left(k^{r+2}-k\right)(k-1)+m r\right]$

and in this case the limit as $m$ and $r \rightarrow \infty$ is $1 / 3$. We then have two double Nörlund matrices which do not give consistent results, although each one is regular. These matrices, of course, are such that the individual columns do not all yield regular transformations.

We next consider the questions of inclusion and equivalence. $Q$ is said to include $P$ if $s_{m n} \rightarrow s(P)$ implies that $s_{m n} \rightarrow(Q), P$ and $Q$ being used for brevity to represent the methods $\left(N, p_{m n}\right)$ and $\left(N, q_{m n}\right)$. If $P$ and $Q$ each include the other, they are said to be equivalent; if $P$ includes $Q$ but is not included in it, $P$ is said to be stronger than $Q$.

If $\left(N, p_{m n}\right)$ and $\left(N, q_{m n}\right)$ are regular, then conditions (3) and the corresponding conditions for $Q$ will hold. Using these conditions for $i=0$ and $j=0$ we see that 


$$
\begin{aligned}
P(x, y) & =\sum P_{m n} x^{m} y^{n}, & Q(x, y) & =\sum Q_{m n} x^{m} y^{n}, \\
p(x, y) & =\sum p_{m n} x^{m} y^{n}, & q(x, y) & =\sum q_{m n} x^{m} y^{n}
\end{aligned}
$$

will all converge for $|x|<1,|y|<1$ and represent analytic functions of $x$ and $y$ in the region indicated. The series

$$
\begin{aligned}
k(x, y) & =\sum k_{m n} x^{m} y^{n}=q(x, y) / p(x, y)=Q(x, y) / P(x, y), \\
l(x, y) & =\sum l_{m n} x^{m} y^{n}=p(x, y) / q(x, y)=P(x, y) / Q(x, y),
\end{aligned}
$$

are convergent for small $x$ and $y$ and

$$
\begin{array}{ll}
\sum_{i, j=0}^{m, n} k_{i j} p_{m-i, n-j}=q_{m n}, & \sum_{i, j=0}^{m, n} k_{i j} P_{m-i, n-j}=Q_{m n}, \\
\sum_{i, j=0}^{m, n} l_{i j} q_{m-i, n-j}=p_{m n}, & \sum_{i, j=0}^{m, n} l_{i j} Q_{m-i, n-j}=P_{m n} .
\end{array}
$$

We are now ready to prove

THEOREM II. If $\left(N, p_{m n}\right)$ and $\left(N, q_{m n}\right)$ are regular, then in order that $\left(N, q_{m n}\right)$ should include $\left(N, p_{m n}\right)$, it is necessary and sufficient that

$$
\left.\sum_{i=0, j=0}^{m, n}\left|k_{i j}\right| P_{m-i, n-j} \leqq H Q_{m n} \quad \text { (H independent of }(m, n)\right),
$$

$$
\begin{aligned}
& \frac{\sum_{j=0}^{n}\left|k_{i j}\right| P_{m-i, n-j}}{Q_{m n}} \rightarrow 0 \quad \text { as }(m, n) \rightarrow(\infty, \infty)(0 \leqq i<m), \\
& \frac{\sum_{i=0}^{m}\left|k_{i j}\right| P_{m-i, n-j}}{Q_{m n}} \rightarrow 0 \quad \text { as }(m, n) \rightarrow(\infty, \infty)(0 \leqq j<n),
\end{aligned}
$$

where it is assumed that $(m-i)$ in (13) and $(n-j)$ in (14) remain fixed as $m$ and $n \rightarrow \infty$.

If we set $s(x, y)=\sum s_{m n} x^{m} y^{n}$, we have for small $x, y$

$$
\sum Q_{m n} N_{m n}^{(q)}(s) x^{m} y^{n}=\sum\left(\sum_{i=0, j=0}^{m, n} q_{m-i, n-j} s_{i j}\right) x^{m} y^{n}=q(x, y) s(x, y),
$$

and similarly

$$
\sum P_{m n} N_{m n}^{(p)}(s) x^{m} y^{n}=p(x, y) s(x, y) .
$$

We have, therefore, 


$$
\begin{aligned}
\sum Q_{m n} N_{m n}^{(q)}(s) x^{m} y^{n} & =\sum k_{m n} x^{m} y^{n} \sum P_{m n} N_{m n}^{(p)}(s) x^{m} y^{n}, \\
Q_{m n} N_{m n}^{(q)}(s) & =\sum_{i=0, j=0}^{m, n} k_{m-i, n-j} P_{i j} N_{i j}^{(p)}(s) .
\end{aligned}
$$

Hence

$$
N_{m n}^{(q)}(s)=\sum_{i=0, j=0}^{m, n} c_{m n i j} N_{i j}^{(p)}(s),
$$

where $c_{m n i j}=k_{m-i, n-j} P_{i j} / Q_{m n}$.

The proof of the theorem is now readily obtained by another application of Theorem VI of Chapter I of S.S.C.F. In view of (15), condition (12) is seen to be equivalent to $\left(\mathrm{A}^{*}\right)_{2}$ and $\left(\mathrm{A}_{1}^{*}\right)_{2}$ of that theorem. From (15) and (10) it is apparent that $\left(\mathrm{C}_{1}^{*}\right)_{2}$ holds, and conditions (13) and (14) are equivalent to conditions $\left(\mathrm{E}_{1}^{*}\right)$ and $\left(\mathrm{E}_{2}{ }^{*}\right)$ of the theorem involved.

We consider finally the question of equivalence and prove

TheORem III. In order that two regular Nörlund methods $\left(N, p_{m n}\right)$ and $\left(N, q_{m n}\right)$ should be equivalent, it is necessary and sufficient that

$$
\sum\left|k_{m n}\right|<\infty, \quad \sum\left|l_{m n}\right|<\infty .
$$

First we prove the necessity. Since $p_{00}>0$ and $q_{00}>0$, it follows that $k_{00}>0$ and $l_{00}>0$. Since $\left(N, q_{m n}\right) \supset\left(N, p_{m n}\right)$, it follows from condition (12) of Theorem II that $k_{00} P_{m n} \leqq H Q_{m n}$. Hence $P_{m n} / Q_{m n}$ remains bounded and in similar fashion we see that $\left(N, p_{m n}\right) \supset\left(N, q_{m n}\right)$ implies that $Q_{m n} / P_{m n}$ remains bounded. If we break off the left-hand side of (12) at the term $i=\mu<m, j=\nu<n$, divide by $P_{m n}$, and allow $m$ and $n$ to become infinite while $\mu$ and $\nu$ remain fixed, we obtain

$$
\sum_{i=0, j=0}^{\mu, \nu}\left|k_{i j}\right| \leqq H \lim \sup \left(Q_{m n} / P_{m n}\right) .
$$

Thus we obtain the first inequality in (16) and the proof of the second one is entirely analogous.

We next prove the sufficiency of the conditions. $\sum\left|k_{m n}\right|<\infty$ implies that $k_{m n}$ and $k_{m n} / Q_{m n}$ tend to zero as $m, n \rightarrow \infty$. Furthermore, from (11),

$$
P_{m n}=\sum_{i=0, j=0}^{m, n} Q_{i j} l_{m-i, n-j} \leqq Q_{m n} \sum\left|l_{m n}\right|
$$

whence 


$$
\sum_{i=0, j=0}^{m, n} P_{m-i, n-i}\left|k_{i j}\right| \leqq Q_{m n} \sum\left|k_{m n}\right| \sum\left|l_{m n}\right| .
$$

This is condition (12) of Theorem II with

$$
H=\sum\left|k_{m n}\right| \sum\left|l_{m n}\right| \text {. }
$$

Likewise, from (17),

$$
\sum_{j=0}^{n} P_{m-i, n-j}\left|k_{i j}\right| \leqq Q_{m-i, n} \sum\left|l_{m n}\right| \sum_{j=0}^{n}\left|k_{i j}\right|
$$

where $(0 \leqq i<m)$. Hence (13) follows from the first equality in (8) and similarly (14) follows from the second equality in (8). Thus we have shown that conditions (16) are sufficient that $\left(N, q_{m n}\right) \supset\left(N, p_{m n}\right)$. An analogous discussion will show that $(16)$ is sufficient that $\left(N, p_{m n}\right)$ $\supset\left(N, q_{m n}\right)$.

Thus the necessity and sufficiency of conditions (16) for the equivalence of $\left(N, p_{m n}\right)$ and $\left(N, q_{m n}\right)$ have been proved.

UNIVERSITY OF CINCINNATI 